\title{
Endovascular Treatment over Standard Medical Therapy in Acute Ischemic Stroke: A Meta-Analysis of Randomized Controlled Trials
}

Paolo Cerrone ${ }^{1}$ and Carmine Marini ${ }^{2 *}$

${ }^{1}$ Department of Biotechnological and Applied Clinical Sciences, University of L'Aquila, Italy

${ }^{2}$ Department of Life, Health and Enviromental Sciences, University of L'Aquila, Italy

\begin{abstract}
Background: Endovascular treatment plays a continuously expanding role in the treatment of acute ischemic stroke. Several randomized controlled clinical trials evaluating possible benefits of endovascular therapy were conducted. From these trials emerged conflicting results. The aim of this study was to evaluate the efficacy of endovascular treatment over standard therapy in acute ischemic stroke.

Methods: We searched in PubMed and EMBASE databases from the date of inception until 2015/07/31. The primary outcome was the functional neurological outcome at 90 days measured with modified Rankin Score $\leq 2$. Secondary outcomes were partial or complete recanalization, assessed with modified arterial occlusive lesion, thrombolysis in myocardial infarction or thrombolysis in cerebral infarction scores of 2-3 and mortality. Safety outcome was Symptomatic Intracranial Hemorrhage (SICH). Data were pooled in the control and intervention groups, and odds ratios were calculated with $95 \%$ interval confidence. Statistical heterogeneity was evaluated with the $X^{2}$ Mantel-Haenszel method and $\mathrm{I}_{2}$ method. A $p$ value $<0.05$ was considered statistically significant. Heterogeneity was considered significant for $P$ values $<0.10$.

Results: 12 trials were included with 2725 participants. Compared with standard therapy, endovascular treatment significantly improved the outcome at 90 days (OR: $1.77 ; 95 \% \mathrm{Cl}: 1.51-2.08$ ). This result was affected by a significative heterogeneity. After a sensitivity analysis, excluding the trials in which the demonstration of large vessel occlusion was not an inclusion criterion, primary outcome was improved in the intervention group (OR: 2.05 95\% $\mathrm{Cl}$ : 1.70-2.46), with non-significant heterogeneity. The recanalization rate was higher and mortality was lower in the intervention groups, but these differences were not significant. The proportion of $\mathrm{SICH}$ was marginally higher in the intervention groups, again without any statistical significance.
\end{abstract}

Conclusion: Endovascular treatment for acute ischemic stroke ensures a significant increase of patients with favorable outcome compared with standard therapy in the absence of risk. Vascular studies before treatment are mandatory.

Keywords: Endovascular treatment; Acute ischemic stroke treatment; Thrombectomy; Intra-arterial thrombolysis

\section{Introduction}

Acute stroke treatment has undergone significant evolution during the past decades. Currently, intravenous thrombolysis using tissue plasminogen activator (IV-tPA) within $4.5 \mathrm{~h}$ of symptom onset is the established treatment for ischemic stroke and is associated with improved functional outcome. Despite the introduction of intravenous thrombolysis with tPA in clinical settings has changed dramatically the outcome of patients, there are several limitations related to this treatment. Strict exclusion criteria make only about $25 \%$ of patients eligible for intravenous thrombolysis. Further, the narrow time window limits the proportion of patients who are treatable [1]. IVtPa is associated with early recanalization in only $21 \%$ of cases of large intracranial vessel occlusion, and the frequency of re-occlusion is about $12 \%[2,3]$. Intravenous administration of tPA often proves ineffective in dissolving thrombi localized in intracranial large vessels, and this may explain the low rates of recanalization after treatment.

Endovascular techniques, both intra-arterial administration of thrombolytic drugs and thrombectomy with special devices, promote dissolution of intracranic large vessels thrombi.

Advantages of endovascular treatment over intravenous thrombolysis may include a selective lytic action into the occluding thrombus, permitting lower amounts of haemorrhagic complications related to systemic concentration of thrombolytic agents [4]. Patients out of time window or with contraindications to intravenous thrombolysis, could be eligible for primary endovascular treatment [5]. On the other hand endovascular treatment is not free of complications. The procedure could require general anesthesia, which can increase the risk of in-hospital mortality [6], and can cause catheter-related complications. Another factor to consider is the need for an experienced interventional neuroradiology team, not available in all stroke centers.

Endovascular treatment benefits have been debated in the last years. Several Randomized Controlled Trials (RCT), comparing endovascular treatments versus standard therapy have been conducted, but these studies showed conflicting results. The wide differences between these RCTs and the relatively small samples recruited made impossible to draw conclusions.

*Corresponding author: Carmine Marini, Department of Life, Health and Enviromental Sciences, University of L'Aquila, Piazza Salvatore Tommasi, 67100 L'Aquila, Italy, Tel: 08624347430862; Fax: 0862368553; E-mail: carmine.marini@univaq.it

Received April 26, 2016; Accepted May 11, 2016; Published May 16, 2016

Citation: Cerrone P, Marini C (2016) Endovascular Treatment over Standard Medical Therapy in Acute Ischemic Stroke: A Meta-Analysis of Randomized Controlled Trials. Cardiovasc Pharm Open Access 5: 181. doi:10.4172/23296607.1000181

Copyright: $\odot 2016$ Cerrone $\mathrm{P}$, et al. This is an open-access article distributed under the terms of the Creative Commons Attribution License, which permits unrestricted use, distribution, and reproduction in any medium, provided the original author and source are credited. 
In the meantime, endovascular techniques have been greatly improved with development of modern generation devices, such as the stentretrievers, and might have contributed to the differences in trial results [7].

In the last years several meta-analysis were conducted in order to determine the real impact of endovascular treatment in acute stroke treatment, but these studies considered only a subset of available RCT's, thus preventing from drawing firm conclusions on the global efficacy of endovascular treatment.

The aim of this study was to evaluate the efficacy of endovascular treatment (intra-arterial thrombolysis and/or thrombectomy) in acute ischemic stroke in the overall population and in specific subsets.

\section{Methods}

\section{Inclusion criteria}

We searched PubMed and EMBASE databases from the date of inception until 2015/07/31 using the terms "ischemic stroke" and "intraarterial" and "thrombolysis" or "thrombectomy" or "endovascular treatment". Reference lists of relevant articles, congress presentations and conference abstracts were manually searched. Studies published in foreign national language for which translation was unavailable, were excluded. We selected only Randomized Controlled Trials (RCTs) which compared endovascular treatment (intra-arterial thrombolysis and/or mechanical thrombectomy) alone or associated to intravenous thrombolysis, with standard treatments (standard care or intravenous thrombolysis). We excluded RCTs in which endovascular treatment was directly compared with intravenous thrombolysis. There were no restrictions in study age, included patient age, imaging criteria, or National Institutes of Health Stroke Scale (NIHSS) score. We excluded studies without a control group and trials in which primary outcome data were not available or could not be extracted from study groups. We extracted the following information: age, National Institutes of Health Stroke Scale (NIHSS) score at baseline, principal inclusion and exclusion criteria, requirement of arterial occlusion for randomization, neuroimaging techniques used, administered therapy. Quality of included trials was formally assessed with the Jadad scale.

\section{Outcomes}

The primary outcome was the proportion of patients with a modified Rankin Score (mRS) of 0-2 at 90 days after the treatment. Secondary outcomes were partial or complete recanalization, assessed with modified Arterial Occlusive Lesion (mAOL) recanalization score, Thrombolysis in Myocardial Infarction (TIMI) score or thrombolysis in cerebral infarction (TICI) score of 2-3, and mortality. Safety outcome was Symptomatic Intracranial Haemorrhage (SICH).

\section{Statistical analysis}

We conducted statistical analysis using SPSS software. We compared outcomes between endovascular treatment and control groups for all RCTs. We used the Mantel-Haenszel (MH) method to calculate the pooled odds ratio and its $95 \%$ confidence interval. Statistical heterogeneity was evaluated with the $\chi^{2}$ Mantel-Haenszel method and with $I_{2}$ method. Heterogeneity was considered significant if the $P$ values of $\chi^{2}$ statistics were $<0.10$. We regarded an $\mathrm{I} 2$ of $<40 \%$ as minimal heterogeneity, $40 \%-75 \%$ as modest heterogeneity, and $>75 \%$ as substantial heterogeneity.

Some degree of heterogeneity was expected and to identify sources of heterogeneity, two subgroup meta-analyses were pre-specified on the base of vessel occlusion status (required demonstrated vessel occlusion for randomization or not) for the primary outcome. We also generated other subgroups considering the type of treatment (RCTs comparing intra-arterial thrombolysis with standard therapy and RCTs comparing thrombectomy with standard therapy).

\section{Results}

A total 1935 articles were identified. 1759 did not match the eligibility criteria. The remaining 176 articles were pertaining to 18 studies available for full text evaluation. Of these 18 studies, 3 were not randomized controlled trials and were thus excluded. For the purpose of the present study 3 studies were further excluded because they compared directly endovascular treatment with intravenous thrombolysis. The characteristics of excluded studies are reported in Table 1. The remaining 12 randomized controlled trials met all the selection criteria and were included in the meta-analysis.

\section{Study characteristics}

The selected trials included in total 2725 participants. Study characteristics are shown in Table 2. Quality of study was high in all cases (Jadad score between 3 to 5).

In four RCTs, patients of the treatment group received exclusively intra-arterial thrombolysis. Intravenous thrombolysis was administered neither in treatment nor in control groups. In the remaining eight studies both intervention groups and control groups received intravenous thrombolysis if recommended, in different proportions (from $<50 \%$ of treated patients in MR RESCUE to $100 \%$ of treated patients in IMS III, EXTEND-IA, SWIFT PRIME) [8-10]. In these studies, endovascular treatment consisted of mechanic thrombectomy plus, eventually, intra-arterial thrombolysis was used.

Time to inclusion from symptoms onset varied between $<3 \mathrm{~h}$ in the IMS III trial to $<24 \mathrm{~h}$ in the AUST trial $[11,12]$. Patients with an occlusion in an artery of anterior circulation were included in ten studies. Three studies (AUST, IMS III and THRACE) included also strokes with occlusion of an artery of posterior circulation (in THRACE were included only patients with occlusion of the upper third of basilary artery) [13]. All studies but one required imaging of vessel occlusion before inclusion. In the IMS III trial, 284 patients had been randomized before CT angiography.

In ESCAPE, EXTEND-IA, SWIFT PRIME and REVASCAT trials, among the inclusion criteria there was also the evaluation of the ischemic core extension $[8,9,14,15]$. Ischemic core extension was indirectly measured in ESCAPE trial and REVASCAT trial applying the ASPECTS score on non-enhanced CT imaging. In EXTEND-IA ischemic core's volume, measured with CT or MR perfusional study, should be $<70 \mathrm{ml}$. In SWIFT PRIME perfusional studies were initially used to determine the extension of ischemic core, but after a protocol revision, perfusional imaging was no longer mandatory.

\begin{tabular}{|c|c|}
\hline Study & Reason for exclusion \\
\hline Ducroq et al. [24] & $\begin{array}{l}\text { This is not a comparison of intra-arterial thrombolysis } \\
\text { versus control, since only the control group is given } \\
\text { intravenous thrombolysis }\end{array}$ \\
\hline $\begin{array}{l}\text { SYNTHESIS PILOT, } \\
2010 \text { [22] }\end{array}$ & $\begin{array}{l}\text { This is a direct comparison of intra-arterial thrombolysis } \\
\text { versus intravenous thrombolysis }\end{array}$ \\
\hline $\begin{array}{l}\text { SYNTHESIS } \\
\text { EXPANSION } 2013\end{array}$ & $\begin{array}{l}\text { This is a direct comparison of intra-arterial thrombolysis } \\
\text { versus intravenous thrombolysis }\end{array}$ \\
\hline
\end{tabular}


Citation: Cerrone P, Marini C (2016) Endovascular Treatment over Standard Medical Therapy in Acute Ischemic Stroke: A Meta-Analysis of Randomized Controlled Trials. Cardiovasc Pharm Open Access 5: 181. doi:10.4172/2329-6607.1000181

Page 3 of 8

\begin{tabular}{|c|c|c|c|c|c|c|c|c|}
\hline Trial & $\begin{array}{l}\text { Inclusion } \\
\text { criteria }\end{array}$ & Imaging techniques & $\begin{array}{c}\text { Time } \\
\text { window }\end{array}$ & Endovascular treatment & $\begin{array}{l}\text { Control } \\
\text { group }\end{array}$ & Recanalization & $\begin{array}{l}\text { Clinical } \\
\text { outcome }\end{array}$ & $\begin{array}{l}\text { Symptomatic } \\
\text { intracranial } \\
\text { Hemhorrage }\end{array}$ \\
\hline PROACT & $\begin{array}{l}\text { MCA occlusion } \\
\text { (M1 o M2); } \\
\text { NIHSS } \geq 4 ; \\
\text { age } 18-85 \text { yr }\end{array}$ & angiography & $<6 \mathrm{~h}$ & $6 \mathrm{mg}$ ia pro-UK+iv heparine & iv heparine & $\begin{array}{l}\text { TIMI 2-3: } 57.7 \% \\
\text { vs. } 14.3 \% \text { in the } \\
\text { control group }\end{array}$ & $\begin{array}{l}\text { Mrs } \leq 1 \text { in } \\
30.8 \% \text { vs. } \\
21.4 \% \text { of } \\
\text { control group }\end{array}$ & $\begin{array}{l}15.4 \% \text { vs. } \\
7.1 \% \text { in the } \\
\text { control group }\end{array}$ \\
\hline PROACT II & $\begin{array}{l}\text { MCA occlusion; } \\
\text { NIHSS } \geq 4 ; \text { age } \\
18-85 \text { yr; }\end{array}$ & angiography & $<6 \mathrm{~h}$ & $9 \mathrm{mg}$ ia pro-UK+iv heparine & iv heparine & $\begin{array}{l}\text { TIMI 2-3: } 66 \% \text { vs. } \\
\text { 18\% of control } \\
\text { group }\end{array}$ & $\begin{array}{c}\mathrm{mRS} \leq 2 \text { in } \\
39.7 \% \text { vs. } \\
25.4 \% \text { of } \\
\text { control group }\end{array}$ & $\begin{array}{c}10 \% \text { vs. } 2 \% \\
\text { in the control } \\
\text { group }\end{array}$ \\
\hline AUST & $\begin{array}{l}\text { occlusion } \\
\text { in posterior } \\
\text { circulation; } \\
\text { age } 18-85 \mathrm{yr}\end{array}$ & angiography & $<24 \mathrm{~h}$ & anticoagulants+ia UK & anticoagulants & ND & $\begin{array}{c}\text { mRS } \leq 2 \text { at } 6 \\
\text { months in } 50 \% \\
\text { vs. } 13 \% \text { of } \\
\text { control group }\end{array}$ & ND \\
\hline MELT & $\begin{array}{l}\text { Occlusion in the } \\
\text { M1 or M2 tract of } \\
\text { MCA; NIHSS }>5 ; \\
\text { age } 20-75 \text { yr }\end{array}$ & angiography & $<6 \mathrm{~h}$ & ia UK & $\begin{array}{l}\text { standard } \\
\text { therapy }\end{array}$ & $\begin{array}{c}\text { partial } \\
\text { recanalization } \\
\geq 50 \%: 47.4 \% \text { in } \\
\text { intervention group }\end{array}$ & $\begin{array}{c}\mathrm{mRS} \leq 2 \text { in } \\
49.1 \% \text { vs. } \\
38.6 \% \text { of } \\
\text { control group }\end{array}$ & $\begin{array}{l}9 \% \text { vs. } 2 \% \text { of } \\
\text { control group }\end{array}$ \\
\hline IMS III & $\begin{array}{l}\text { NIHSS } \geq 10 \\
\text { age } 18-82 \mathrm{yr}\end{array}$ & $\begin{array}{l}\text { CTA only in subjects } \\
\text { with NIHSS }=8-9\end{array}$ & $\begin{array}{c}<3 \mathrm{~h} \text { for iv } \\
\text { thrombolysis } \\
<5 \text { hours for } \\
\text { endovascular } \\
\text { treatment }\end{array}$ & $\begin{array}{l}\text { iv thrombolysis+ia } \\
\text { thromboysis/thrombectomy }\end{array}$ & $\begin{array}{c}\text { iv } \\
\text { thrombolysis }\end{array}$ & $\begin{array}{l}\mathrm{TICI} 2-3: 75 \% \\
\mathrm{TICl} 2 \mathrm{~b}-3: 41 \% \\
\text { (intervention } \\
\text { group) }\end{array}$ & $\begin{array}{c}\mathrm{mRS} \leq 2 \text { in } \\
40.8 \% \text { vs. } \\
38.7 \% \text { control } \\
\text { group }\end{array}$ & $\begin{array}{c}6.2 \% \text { vs. } \\
5.9 \% \text { of } \\
\text { control group }\end{array}$ \\
\hline $\begin{array}{c}\text { MR } \\
\text { RESCUE }\end{array}$ & $\begin{array}{l}\text { MCA occlusion } \\
\text { in M1 e M2 and } \\
\text { distal ICA } \\
\text { NIHSS } 6-29 \\
\text { age } 18-85 \mathrm{yr}\end{array}$ & $\begin{array}{c}\text { CTA/MRA or } \\
\text { angiography;perfusional } \\
\text { CT/MR }\end{array}$ & $<8 \mathrm{~h}$ & $\begin{array}{c}\text { embolectomy (MERCl or } \\
\text { PENUMBRA) }\end{array}$ & $\begin{array}{l}\text { standard } \\
\text { therapy }\end{array}$ & $\begin{array}{c}\mathrm{TICl} 2-3: 71.4 \% \text { vs. } \\
86.7 \% \text { of control } \\
\text { group }\end{array}$ & $\begin{array}{c}\mathrm{mRS} \leq 2 \text { in } \\
12.5 \% \text { vs. } \\
18.5 \% \text { of } \\
\text { control group }\end{array}$ & $\begin{array}{c}4.7 \% \text { vs. } \\
3.7 \% \text { of } \\
\text { control group }\end{array}$ \\
\hline MR CLEAN & $\begin{array}{c}\text { MCA occlusion } \\
\text { in M1 o M2 tract, } \\
\text { A1 or A2 tract of } \\
\text { ACA; } \\
\text { NIHSS } \geq 2 \\
\text { age }>18 \text { yr }\end{array}$ & $\begin{array}{l}\text { CTA/MRA or } \\
\text { angiography }\end{array}$ & $<6 \mathrm{~h}$ & $\begin{array}{l}\text { ia thrombolysis (rt-PA or } \\
\text { UK) and/or thrombectomy } \\
\text { (eventually preceded by iv } \\
\text { thrombolysis) }\end{array}$ & $\begin{array}{l}\text { standard } \\
\text { therapy } \\
\text { (also iv } \\
\text { thrombolysis) }\end{array}$ & $\begin{array}{l}\mathrm{TICl} 2 \mathrm{~b}-3: 58.7 \% \\
\text { of control group }\end{array}$ & $\begin{array}{c}\mathrm{mRS} \leq 2 \text { in } \\
32.6 \% \text { vs. } \\
19.1 \% \text { of } \\
\text { control group }\end{array}$ & $\begin{array}{c}7.7 \% \text { vs. } \\
6.4 \% \text { of } \\
\text { control group }\end{array}$ \\
\hline ESCAPE & $\begin{array}{c}\text { occlusion in MCA } \\
\text { and distal ICA } \\
\text { age }>18 \mathrm{yr} \\
\text { ASPECT }>6\end{array}$ & $\begin{array}{c}\text { CTA; } \\
\text { ASPECTS score }\end{array}$ & $<12 \mathrm{~h}$ & $\begin{array}{l}\text { standard therapy (also } \\
\text { iv thrombolysis in } \\
72.7 \% \text { )+thrombectomy }\end{array}$ & $\begin{array}{l}\text { standard } \\
\text { therapy } \\
\text { (also iv } \\
\text { thrombolysis) }\end{array}$ & $\begin{array}{l}\mathrm{TICl} 2 \mathrm{~b}-3: 72.4 \% \\
\text { vs. AOL: } 31.2 \% \text { in } \\
\text { the control group }\end{array}$ & $\begin{array}{c}\mathrm{mRS} \leq 2 \text { in } \\
53.0 \% \text { vs. } \\
29.3 \% \text { of } \\
\text { control group }\end{array}$ & $\begin{array}{c}3.6 \% \text { vs. } \\
2.7 \% \text { of } \\
\text { control group }\end{array}$ \\
\hline EXTEND-IA & $\begin{array}{c}\text { occlusion in MCA } \\
\text { and distal ICA } \\
\text { age }>18 \mathrm{yr}\end{array}$ & $\begin{array}{c}\text { CTA/MRA; } \\
\text { perfusional } \mathrm{CT} / \mathrm{MR} \\
\text { (core<70 mL) }\end{array}$ & $\begin{array}{l}<4,5 \mathrm{~h} \text { for iv } \\
\text { thrombolysis } \\
\quad<6 \mathrm{~h} \text { for } \\
\text { endovascular } \\
\text { treatment }\end{array}$ & $\begin{array}{c}\text { iv } \\
\text { thrombolysis+thrombectomy } \\
\text { with SOLITAIRE }\end{array}$ & iv & $\begin{array}{l}\text { TIMI 2-3: } 94.0 \% \\
\text { vs. } 43.0 \% \text { in the } \\
\text { control group }\end{array}$ & $\begin{array}{c}\mathrm{mRS} \leq 2 \text { in } \\
71.0 \% \text { vs. } \\
40.0 \% \text { of } \\
\text { control group }\end{array}$ & $\begin{array}{l}0 \% \text { vs. } 6.0 \% \\
\text { of control } \\
\text { group }\end{array}$ \\
\hline $\begin{array}{l}\text { SWIFT } \\
\text { PRIME }\end{array}$ & $\begin{array}{l}\text { occlusion of MCA } \\
\text { or distal ICA; } \\
\text { age } 18-85 \mathrm{yr}\end{array}$ & $\begin{array}{l}\text { CTA or MRA; } \\
\text { perfusional CT/MR or } \\
\text { ASPECTS score }\end{array}$ & $<6 \mathrm{~h}$ & $\begin{array}{c}\text { iv } \\
\text { thrombolysis+thrombectomy } \\
\text { with SOLITAIRE }\end{array}$ & $\begin{array}{l}\text { iv } \\
\text { thrombolysis }\end{array}$ & TICI 2b-3: 88\% & $\begin{array}{c}\text { mRS } \leq 2 \text { nel } \\
60.2 \% \text { vs. } \\
34.7 \% \text { in the } \\
\text { control group }\end{array}$ & $\begin{array}{l}0 \% \text { vs. } 3 \% \\
\text { in the control } \\
\text { group }\end{array}$ \\
\hline REVASCAT & $\begin{array}{c}\text { occlusion of M1 } \\
\text { tract of MCA or } \\
\text { ICA; } \\
\text { NIHSS }>6 \\
\text { age } 18-80 \mathrm{yr} \\
\text { ASPECTS }>7\end{array}$ & $\begin{array}{c}\text { CTA/MRA; } \\
\text { ASPECTS score }\end{array}$ & $<8 \mathrm{~h}$ & $\begin{array}{l}\text { thrombectomy with } \\
\text { SOLITAIRE also after iv } \\
\text { thrombolysis }(68 \%)\end{array}$ & $\begin{array}{l}\text { Standard } \\
\text { therapy } \\
\text { (also iv } \\
\text { thrombolysis) } \\
\quad(77.7 \%)\end{array}$ & $\begin{array}{l}\mathrm{TICl} 2-3: 90.2 \% \\
\mathrm{TICl} 2 \mathrm{~b}-3: 65.7 \%\end{array}$ & $\begin{array}{l}\mathrm{mRS} \leq 2 \text { in } \\
43.7 \% \text { vs. } \\
28.2 \% \text { in the } \\
\text { control group }\end{array}$ & $\begin{array}{l}4.9 \% \text { vs. } \\
1.9 \% \text { in the } \\
\text { control group }\end{array}$ \\
\hline THRACE & $\begin{array}{l}\text { occlusion of the } \\
\text { intracranial ICA, } \\
\text { M1 tract of MCA, } \\
\text { upper third of } \\
\text { BA; } \\
\text { NIHSS10-25 }\end{array}$ & & $<4 \mathrm{~h}$ & $\begin{array}{l}\text { iv thrombolysis+mechanic } \\
\text { thrombectomy }\end{array}$ & $\begin{array}{l}\text { iv } \\
\text { thrombolysis }\end{array}$ & & $\begin{array}{c}\mathrm{mRS} \leq 2 \text { in } \\
54.2 \% \mathrm{vs} . \\
42.1 \% \\
\text { of control group }\end{array}$ & \\
\hline
\end{tabular}

Table 2: Characteristics of included trials.

\section{Primary and secondary outcomes}

Data concerning the proportion of patients with Mrs $\leq 2$ at 90 days was available in all the 11 trials (Figure 1). The proportion of patients with mRS $0-2$ after 90 days was $44.6 \%$ in the intervention group and $31.4 \%$ in the control group. The outcome favored the intervention group with an odds ratio of 1.77 (95\% CI: 1.51-2.08). However, the result was affected by a significant heterogeneity $\left(\chi_{2}=19.81 ; I_{2}=50 \%\right.$;
$\mathrm{P}=0.03$ ). Figure 2 reports a sensitivity analysis excluding RCTs in which there was not a mandatory use of neuroimaging techniques to identify patients with a large artery occlusion before randomization. The proportion of patients with a good outcome (mRS $\leq 2)$ was $45.4 \%$ in the treatment group and $29.5 \%$ in the control group. The outcomes for the intervention groups improved, with an odds ratio of 2.05 (95\% CI: 1.70-2.46), without any significant heterogeneity $\left(\chi_{2}=9.96 ; I_{2}=10 \%\right.$; $\mathrm{P}=0.03)$. 
Citation: Cerrone P, Marini C (2016) Endovascular Treatment over Standard Medical Therapy in Acute Ischemic Stroke: A Meta-Analysis of Randomized Controlled Trials. Cardiovasc Pharm Open Access 5: 181. doi:10.4172/2329-6607.1000181

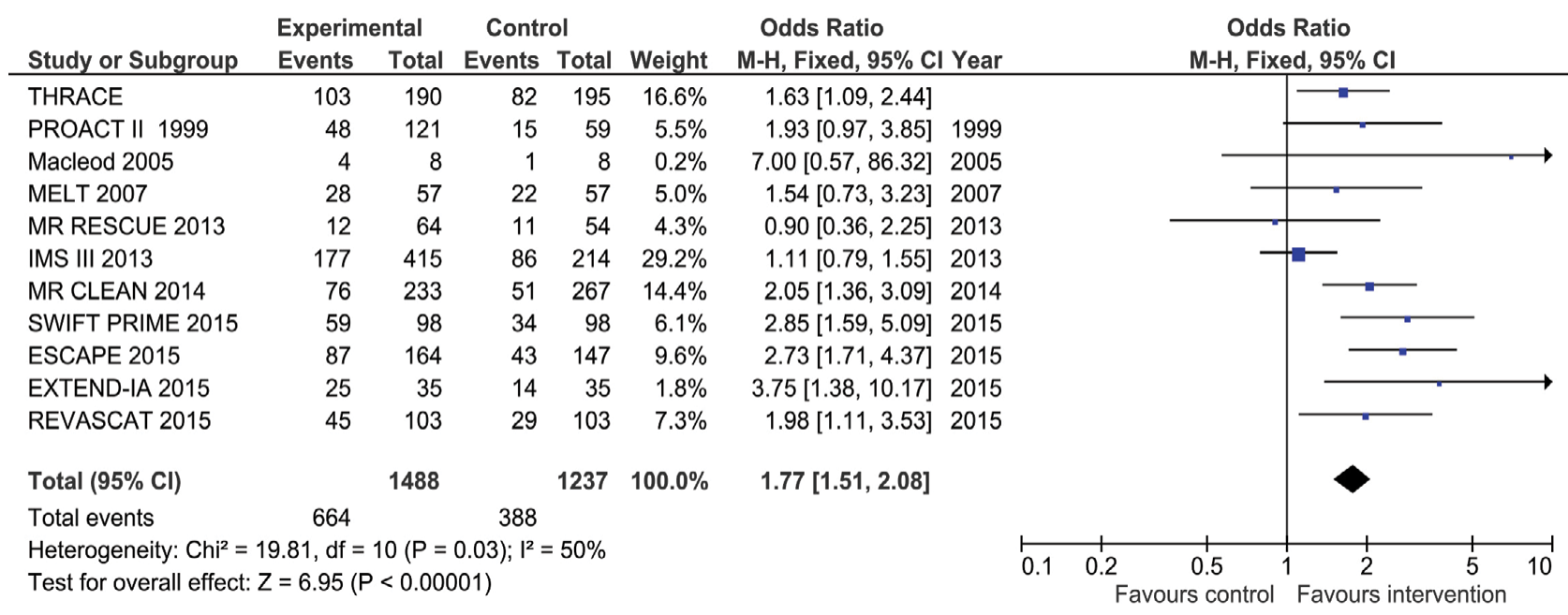

Figure 1: Effectiveness of endovascular treatment on functional independence (modified Rankin Scale [mRS] $\leq 2$ ) at 90 days.

\begin{tabular}{|c|c|c|c|c|c|c|c|c|c|c|c|}
\hline Study or Subgroup & \multicolumn{2}{|c|}{ Experimental } & Control & $\begin{array}{l}\text { ol } \\
\text { Total }\end{array}$ & Weight & \multicolumn{2}{|l|}{ Odds Ratio } & \multicolumn{4}{|c|}{$\begin{array}{c}\text { Odds Ratio } \\
\text { M-H, Fixed, 95\% Cl }\end{array}$} \\
\hline THRACE & 103 & 190 & 82 & 195 & $23.5 \%$ & $1.63[1.09,2.44]$ & & & & $\longrightarrow$ & \\
\hline PROACT II 1999 & 48 & 121 & 15 & 59 & $7.7 \%$ & $1.93[0.97,3.85]$ & 1999 & & & & \\
\hline Macleod 2005 & 4 & 8 & 1 & 8 & $0.3 \%$ & $7.00[0.57,86.32]$ & 2005 & & & & \\
\hline MELT 2007 & 28 & 57 & 22 & 57 & $7.1 \%$ & $1.54[0.73,3.23]$ & 2007 & & & & \\
\hline MR RESCUE 2013 & 12 & 64 & 11 & 54 & $6.1 \%$ & $0.90[0.36,2.25]$ & 2013 & & & & \\
\hline MR CLEAN 2014 & 76 & 233 & 51 & 267 & $20.3 \%$ & $2.05[1.36,3.09]$ & 2014 & & & & \\
\hline REVASCAT 2015 & 45 & 103 & 29 & 103 & $10.3 \%$ & $1.98[1.11,3.53]$ & 2015 & & & & \\
\hline ESCAPE 2015 & 87 & 164 & 43 & 147 & $13.5 \%$ & $2.73[1.71,4.37]$ & 2015 & & & & \\
\hline SWIFT PRIME 2015 & 59 & 98 & 34 & 98 & $8.6 \%$ & $2.85[1.59,5.09]$ & 2015 & & & & \\
\hline EXTEND-IA 2015 & 25 & 35 & 14 & 35 & $2.5 \%$ & $3.75[1.38,10.17]$ & 2015 & & & & \\
\hline Total $(95 \% \mathrm{Cl})$ & & 1073 & & 1023 & $100.0 \%$ & $2.05[1.70,2.46]$ & & & & & \\
\hline Total events & 487 & & 302 & & & & & & & & \\
\hline $\begin{array}{l}\text { Heterogeneity: } \mathrm{Chi}^{2}= \\
\text { Test for overall effect: }\end{array}$ & $\begin{array}{l}.96, \mathrm{df}=\mathrm{s} \\
=7.59(\mathrm{P}\end{array}$ & $\begin{array}{l}P=0.3 \\
<0.000\end{array}$ & $\begin{array}{l}35) ;\left.\right|^{2}=1 \\
01)\end{array}$ & & & & & $\begin{array}{ll}0.1 & 0.2\end{array}$ & 0.5 & 12 & 5 \\
\hline
\end{tabular}

Figure 2: Effectiveness of endovascular treatment on functional independence (modified Rankin Scale [mRS] $\leq 2$ ) at 90 days in in patients with proved Large Vessel Occlusion (LVO)

\begin{tabular}{|c|c|c|c|c|c|c|c|c|c|c|c|}
\hline Study or Subgroup & \multicolumn{2}{|c|}{ Experimental } & \multicolumn{2}{|c|}{ Control } & \multicolumn{3}{|c|}{ Odds Ratio } & \multicolumn{4}{|c|}{$\begin{array}{c}\text { Odds Ratio } \\
\text { M-H, Fixed, } 95 \% \mathrm{Cl}\end{array}$} \\
\hline PROACT II 1999 & 48 & 121 & 15 & 59 & $51.0 \%$ & $1.93[0.97,3.85]$ & 1999 & & & & \\
\hline Macleod 2005 & 4 & 8 & 1 & 8 & $2.1 \%$ & $7.00[0.57,86.32]$ & 2005 & & & & \\
\hline MELT 2007 & 28 & 57 & 22 & 57 & $46.9 \%$ & $1.54[0.73,3.23]$ & 2007 & & & & \\
\hline Total $(95 \% \mathrm{Cl})$ & & 186 & & 124 & $100.0 \%$ & $1.85[1.13,3.03]$ & & & & & \\
\hline Total events & 80 & & 38 & & & & & & & & \\
\hline $\begin{array}{l}\text { Heterogeneity: } \mathrm{Chi}^{2}= \\
\text { Test for overall effect: }\end{array}$ & $\begin{array}{l}33, \mathrm{df}=2 \\
=2.45(\mathrm{P}\end{array}$ & $\begin{array}{l}(P=0.5 \\
=0.01)\end{array}$ & 51); $I^{2}=0$ & & & & & 0.05 & $\begin{array}{c}0.2 \\
\text { Favours control }\end{array}$ & 1 Favours int & $\begin{array}{ll}1 & 1 \\
5 & 20 \\
\text { tervention } & \end{array}$ \\
\hline
\end{tabular}

Figure 3: Effectiveness of intra-arterial thrombolysis on functional independence (modified Rankin Scale [mRS] $\leq 2$ ) at 90 days.

We also analyzed the efficacy of intra-arterial thrombolysis and mechanic thrombectomy developing two different subgroups on the base of the type of intervention. In the subgroup of patients treated with intra-arterial thrombolysis (Figure 3 ) there was a non-significant trend which favored the intervention $(\mathrm{OR}=1.85 ; 95 \% \mathrm{CI}: 1.13-3.03 ; \mathrm{P}=0.01)$. In the subgroup of patients treated with mechanic thrombectomy (Figure 4) a superiority of the intervention groups was observed for the primary outcome $(\mathrm{OR}=2.17 ; 95 \% \mathrm{CI}: 1.77-2.67 ; \mathrm{P}<0.00001)$.

The recanalization rate was higher in the intervention group (Figure 5) with an odds ratio of 4.32 (95\% CI: 3.26-5.72; $\mathrm{P}<0.00001$ ).
There was an increased proportion of patients with symptomatic intracranial hemorrhage in the intervention group (5.9\% vs. $4.3 \%)$, with an odds ratio of 1.34 (95\% CI: 0.92-1.95) but the difference was not statistically significant (Figure 6). Mortality was lower in the intervention $(17.8 \%$ vs. $20.7 \%)$ with an odds ratio of 0.82 (95\% CI: 0.66-1.01) than in control groups although the difference did not reach statistical significance $(\mathrm{P}=0.07)$ (Figure 7$)$.

\section{Discussion}

This meta-analysis evaluated the efficacy of endovascular treatment 
Citation: Cerrone P, Marini C (2016) Endovascular Treatment over Standard Medical Therapy in Acute Ischemic Stroke: A Meta-Analysis of Randomized Controlled Trials. Cardiovasc Pharm Open Access 5: 181. doi:10.4172/2329-6607.1000181

Page 5 of 8

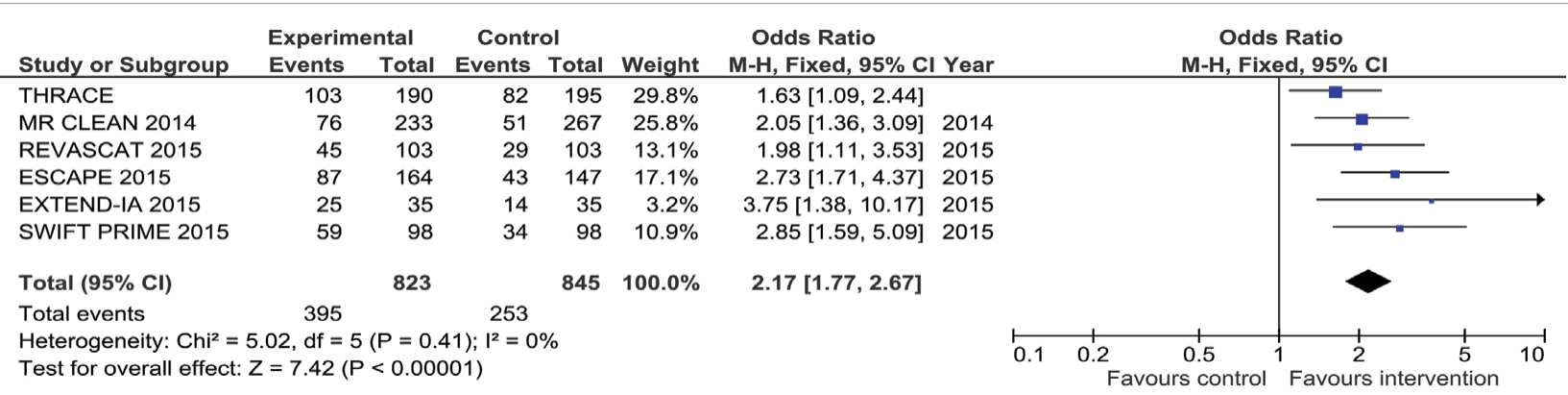

Figure 4: Effectiveness of mechanic thrombectomy on functional independence (modified Rankin Scale [mRS] $\leq 2$ ) at 90 days.

\begin{tabular}{|c|c|c|c|c|c|c|c|c|c|c|c|}
\hline Study or Subgroup & \multicolumn{2}{|c|}{ Experimental } & \multicolumn{2}{|c|}{ Control } & \multicolumn{3}{|c|}{ Odds Ratio } & \multicolumn{4}{|c|}{$\begin{array}{c}\text { Odds Ratio } \\
\text { M-H, Fixed, } 95 \% \mathrm{Cl}\end{array}$} \\
\hline PROACT 1998 & 15 & 26 & 2 & 14 & $2.2 \%$ & $8.18[1.51,44.21]$ & 1998 & & & & \\
\hline PROACT II 1999 & 71 & 108 & $\overline{9}$ & 50 & $8.6 \%$ & $8.74[3.84,19.92]$ & 1999 & & & & \\
\hline MR RESCUE 2013 & 40 & 56 & 39 & 45 & $25.1 \%$ & $0.38[0.14,1.08]$ & 2013 & & & & \\
\hline MR CLEAN 2014 & 157 & 187 & 119 & 207 & $36.8 \%$ & $3.87[2.40,6.24]$ & 2014 & & & & \\
\hline ESCAPE 2015 & 113 & 156 & 43 & 138 & $25.6 \%$ & $5.81[3.51,9.60]$ & 2015 & & & & \\
\hline EXTEND-IA 2015 & 33 & 35 & 15 & 35 & $1.7 \%$ & $22.00[4.55,106.43]$ & 2015 & & & & \\
\hline Total $(95 \% \mathrm{Cl})$ & & 568 & & 489 & $100.0 \%$ & $4.32[3.26,5.72]$ & & & & & \\
\hline Total events & 429 & & 227 & & & & & & & & \\
\hline $\begin{array}{l}\text { Heterogeneity: } \mathrm{Chi}^{2}= \\
\text { Test for overall effect: }\end{array}$ & $\begin{array}{l}9.90, \mathrm{df}= \\
=10.22\end{array}$ & $\begin{array}{l}5(P<0 \\
P<0.0 C\end{array}$ & $\begin{array}{l}.0001) ; 1^{2} \\
001)\end{array}$ & $=83 \%$ & & & & 0.05 & $\begin{array}{l}0.2 \\
\text { Favours control }\end{array}$ & 1 Favours in & $\begin{array}{l}5 \\
\text { intervention }\end{array}$ \\
\hline
\end{tabular}

Figure 5: Effectiveness of endovascular treatment on partial or complete recanalization (modified Arterial Occlusive Lesion [mAOL] recanalization score, Thrombolysis in Myocardial Infarction [TIMI] score or Thrombolysis in Cerebral Infarction [TICI] score of 2-3).

\begin{tabular}{|c|c|c|c|c|c|c|c|c|c|c|}
\hline Study or Subgroup & \multicolumn{2}{|c|}{ Intervention } & \multicolumn{2}{|c|}{ Control } & \multicolumn{3}{|c|}{ Odds Ratio } & \multicolumn{3}{|c|}{$\begin{array}{c}\text { Odds Ratio } \\
\text { M-H, Fixed, 95\% Cl }\end{array}$} \\
\hline PROACT 1998 & 4 & 26 & 1 & 14 & $2.3 \%$ & $2.36[0.24,23.48]$ & 1998 & & & \\
\hline PROACT II 1999 & 11 & 108 & 1 & 54 & $2.5 \%$ & $6.01[0.76,47.84]$ & 1999 & & & \\
\hline Macleod 2005 & 0 & 8 & 0 & 8 & & Not estimable & 2005 & & & \\
\hline MELT 2007 & 5 & 57 & 1 & 57 & $1.9 \%$ & $5.38[0.61,47.63]$ & 2007 & & & \\
\hline IMS III 2013 & 27 & 434 & 13 & 222 & $33.7 \%$ & $1.07[0.54,2.11]$ & 2013 & & & \\
\hline MR RESCUE 2013 & 3 & 64 & 2 & 54 & $4.3 \%$ & $1.28[0.21,7.95]$ & 2013 & & & \\
\hline MR CLEAN 2014 & 18 & 233 & 17 & 267 & $30.5 \%$ & $1.23[0.62,2.45]$ & 2014 & & - & \\
\hline EXTEND-IA 2015 & 0 & 35 & 2 & 35 & $5.1 \%$ & $0.19[0.01,4.08]$ & 2015 & & & \\
\hline REVASCAT 2015 & 5 & 103 & 2 & 103 & $4.0 \%$ & $2.58[0.49,13.59]$ & 2015 & & & \\
\hline SWIFT PRIME 2015 & 0 & 98 & 3 & 98 & $7.3 \%$ & $0.14[0.01,2.72]$ & 2015 & & & \\
\hline ESCAPE 2015 & 6 & 165 & 4 & 150 & $8.4 \%$ & $1.38[0.38,4.98]$ & 2015 & & & \\
\hline Total $(95 \% \mathrm{Cl})$ & & 1331 & & 1062 & $100.0 \%$ & $1.34[0.92,1.95]$ & & & & \\
\hline Total events & 79 & & 46 & & & & & & & \\
\hline $\begin{array}{l}\text { Heterogeneity: } \mathrm{Chi}^{2}= \\
\text { Test for overall effect }\end{array}$ & $\begin{array}{l}69, \mathrm{df}= \\
=1.50\end{array}$ & $\begin{array}{l}(P=0 . \\
=0.13\end{array}$ & $47) ; 1^{2}=c$ & & & & & $\begin{array}{l}0.05 \quad 0.2 \\
\quad \text { Favours intervention }\end{array}$ & 1 Favours C & $\begin{array}{l}5 \\
\text { ntrol }\end{array}$ \\
\hline
\end{tabular}

Figure 6: Risk of Symptomatic Intracranial Hemorrhage $(\mathrm{SICH})$ associated with endovascular treatment.

over standard therapy, either including IV thrombolysis or not, in patients with acute ischemic stroke. The results confirmed that endovascular treatment is highly effective in these patients. This result was even sounder considering the high quality of all included trials.

The most important feature of this meta-analysis was the inclusion of virtually all published studies. The main limitation was unavailability of the original single case data that hampered the execution of some important comparisons such as those on the efficacy of endovascular procedures when performed as a primary intervention and on the usefulness of additional studies of ischemic core extension and collateral circle patency.

Some meta-analyses evaluating the efficacy of endovascular treatment have been published during years, showing different results.

A Cochrane Collaboration review published by O' Rourke et al.
[16] in 2010 analyzed the efficacy of intra-arterial thrombolysis over standard therapy, showing a significative improved outcome in the interventional group. In this study only the first RCTs evaluating the efficacy of intra-arterial thrombolysis were included, but there is no evaluation of more recent RCTs using mechanic thrombectomy. Fields et al. [17] in 2011 considered only RCTs applying intra-arterial thrombolysis in patients with an occlusion of middle cerebral artery, confirming the superiority of intra-arterial thrombolysis. Fargen et al. [18] in 2015 published a meta-analysis including RCTs evaluating the efficacy of intra-arterial thrombolysis and mechanic thrombectomy over standard therapy, with an improved outcome in intervention group. Delgado et al. [19] in 2015 included only recent RCTs, in which mechanic thrombectomy was the only treatment administered in the intervention group. The outcome was significatively favored in the intervention group. Chen et al. [20] in 2015 included in their metaanalysis endovascular treatment RCTs from 2013 to 2015, excluding 


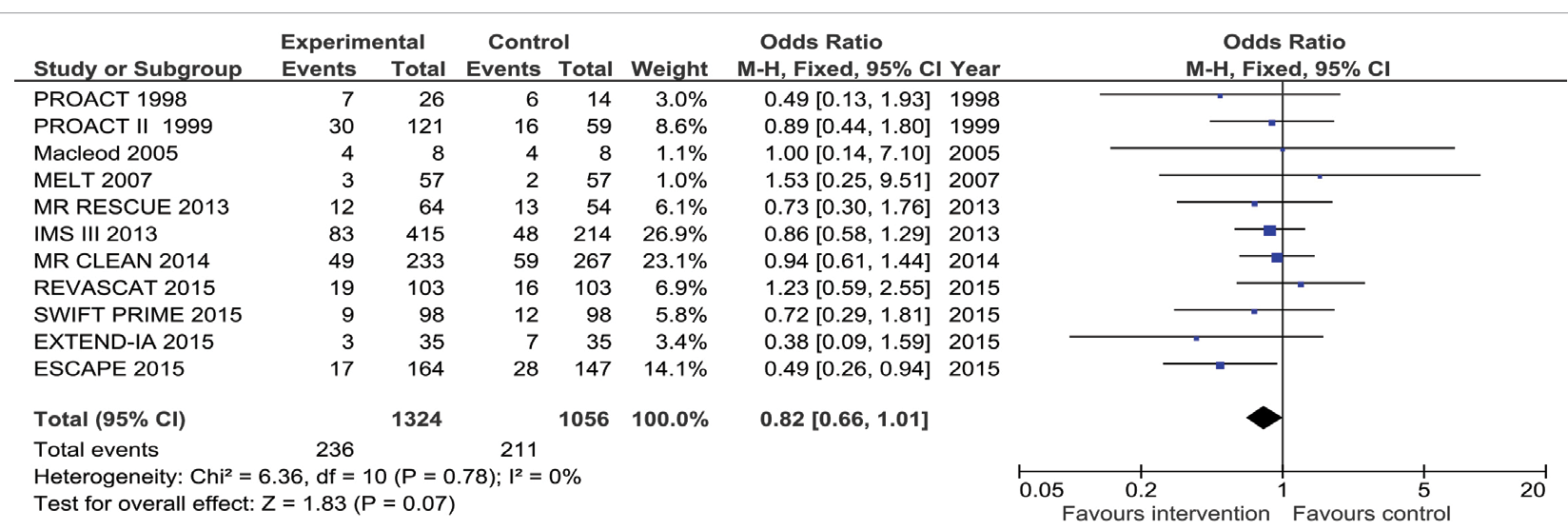

Figure 7: Risk of mortality associated with endovascular treatment

trials which compared intra-arterial thrombolysis with standard therapy. The authors stratified results by Large Vessel Occlusion (LVO) criteria and by use of stent retriever device. They observed an improved outcome in the intervention group considering RCTs with LVO confirmation and RCTs with the use of stent retrievers in the intervention groups. Hong et al. [21] published a systematic review in 2015, including endovascular treatment RCTs from 1998 to 2015, demonstrating the efficacy of endovascular treatment over standard therapy. Previous meta-analyses underlined the efficacy of mechanic thrombectomy or intra-arterial thrombolysis, focusing only on a subgroup of endovascular techniques. The systematic review published by Hong et al. [21] in 2015 included RCTs which compared intraarterial thrombolysis and/or thrombectomy with standard therapy. This study included all the main RCTs conducted to evaluate the efficacy of endovascular treatment (intra-arterial thrombolysis and mechanic thrombectomy) over standard therapy but three RCTs (Synthesis Pilot 2010 [22], SYNTHESIS EXPANSION 2013 [23] and Ducroq et al. [24]) did not compare endovascular treatment with standard medical therapy, but with intravenous thrombolysis, that is not administered in the intervention groups [22-24].

Compared with previous studies, our meta-analysis has the advantage of considering both RCTs evaluating intra-arterial thrombolysis [12,25-27] and RCTs evaluating mechanic thrombectomy [8-11,13-15,28] giving a global point of view of the efficacy and safety of endovascular treatment over standard therapy. We did not included RCTs comparing directly endovascular treatment with intravenous thrombolysis. Qualities of data have been formally evaluated. Sensitivity analyses deemed heterogeneity of studies as negligible and allowed definite conclusions on the effectiveness of endovascular treatment in acute ischemic stroke. The efficacy of endovascular treatment was corroborated by clear trends in more frequent recanalization and in reduced mortality. On the other hand the marginally increased risk of symptomatic intracranial hemorrhage was not significant.

Our results showed that endovascular treatment ensures a higher proportion of patients with a $\mathrm{mRS} \leq 2$ at 90 days compared with standard therapy. However the analysis showed a significant heterogeneity. A sensitivity analysis was done, excluding the RCTs in which intracranial arterial occlusion demonstration on vascular imaging was not an inclusion criterion. Heterogeneity became negligible and the efficacy of endovascular treatment was remarked. The only source of heterogeneity in our study was thus represented by the lack of the demonstration of vascular occlusion before patients' selection. Our results suggest that endovascular treatment should not be performed in patients with acute ischemic stroke if vascular studies have not detected an intracranial large vessel occlusion or has not been performed.

The negligible heterogeneity that remained after exclusion of trials without vascular studies before randomization indicates that the general result of efficacy of endovascular treatments apply to all studies, irrespective of endovascular techniques that were used and of standard treatment that was used in controls.

The benefit was obvious in the subgroup of patients treated with mechanic thrombectomy but the data were consistent for the subgroup of patients treated with intra-arterial thrombolysis also, although not achieving statistical significance. However, urokinase is no longer available; thus mechanical thrombectomy, either in combination with IA trombolytics or not, should be considered the preferred method.

Higher proportions of recanalization were achieved after endovascular treatment, but the results did not reach statistical significance. However, this result is consistent across all studies and may contribute to explain the greater effectiveness of endovascular treatment over standard therapy.

Our study confirmed a trend toward increased risk of SICH in subjects with endovascular treatment confirming the result of other studies [16-19]. Since we evaluated the effectiveness of endovascular treatment on the top of standard therapy, this finding is not surprising and may be linked to the reperfusion of the ischemic area, in which there is a blood-brain-barrier alteration triggered by ischemia [29]. On the other hand, like in most reperfusion studies in stroke patients, this increased risk did not offset the positive effect of the treatment. Endovascular treatment may thus be considered safe enough to be recommended as a valuable intervention when indicated.

A great proportion of treated cases also had IV thrombolysis. However, we were unable to evaluate the interaction between IV thrombolysis and endovascular treatment because of lack of information in almost all published studies. The SYNTHESYS EXPANSION study directly compared endovascular treatment and systemic thrombolysis, but in this study vascular studies before randomization were not performed and probably very few cases had a true indication to endovascular treatment since very few devices were used. Therefore, whether endovascular treatment should be preceded by systemic thrombolysis, when indicated, or may be used as a primary intervention instead of IV rt-PA remains an unanswered question.

There is a great difference in the design and inclusion criteria of the first generation studies, which tested the efficacy of intra-arterial 
thrombolysis and of the last generation studies, which used in almost all cases mechanic thrombectomy. Even in the field of mechanic thrombectomy there can be seen an evolution with gradual replacement of first generation devices with newer ones as stent-retrievers. In RCTs which used mechanic thrombectomy in the intervention groups, there were different proportions of patients treated with stent-retrievers between trials.

The time limit from symptoms onset and groin puncture recommended in the principal international guidelines is 6 hours for anterior circulation strokes and up to $12 \mathrm{~h}$ for basilar artery occlusions. However, only four of the clinical trials included in our meta-analysis, randomized patients up to 8 or $12 \mathrm{~h}$ from symptoms onset and only AUST, IMS III and THRACE included strokes of the posterior circulation therefore, an analysis of time window and of vascular district could not be performed. Effectiveness, beyond the $6 \mathrm{~h}$ time window, even in the posterior circulation has still insufficient support.

The endovascular techniques varied widely across time and studies as well as the devices that have greatly improved with time. It would have been interesting to analyze the impact of these and other aspects on the efficacy of endovascular treatment, but unavailability of data in the published reports prevented these analyses. Still unclear is also the precise role and utility of CTA or MRA diffusion/perfusion

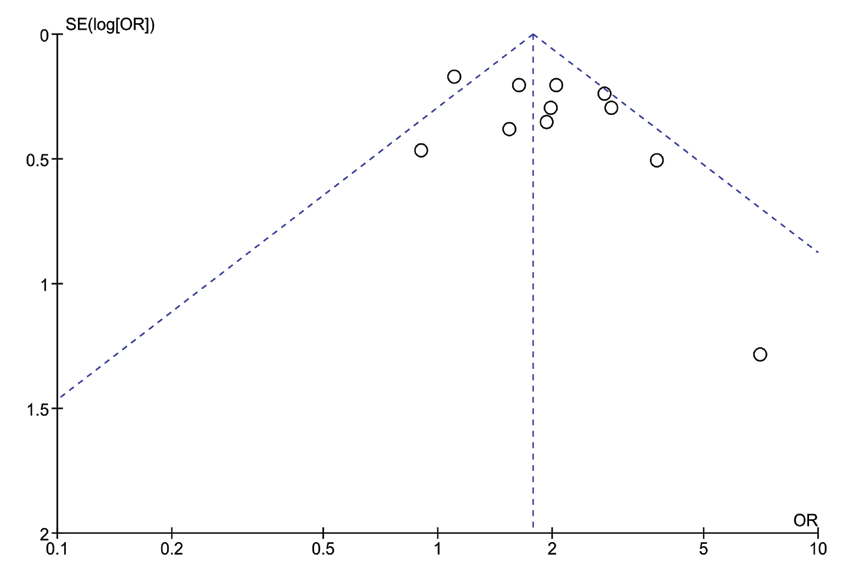

Figure 8: Funnel plot of odds ratios for functional independence (modified Rankin Scale $[\mathrm{mRS}] \leq 2$ ) at 90 days.

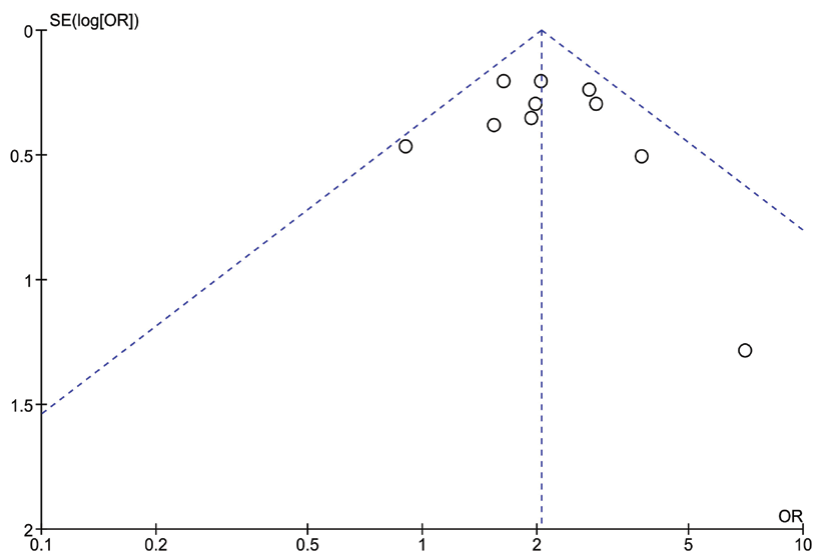

Figure 9: Funnel plot of odds ratios for functional independence (modified Rankin Scale $[\mathrm{mRS}] \leq 2$ ) at 90 days in RCTs assessing Large Vessel Occlusion (LVO). imaging in the selection of patients who will reasonably be responders to endovascular treatment. Lastly, we have to consider that the generalizability of the results is not so obvious, since the included patients differed from those usually seen in a real life setting, because of the strict selection criteria and of the small proportions of admission. Therefore, further studies are needed to address these questions.

In conclusion this meta-analysis showed that patients with an arterial occlusion treated with endovascular treatment showed an improved functional outcome after 3 months from stroke onset compared to patients receiving standard therapy in the absence of significant complications. Vascular studies before treatment are mandatory (Figures 8 and 9).

\section{References}

1. Boode B, Welzen V, Franke C, van Oostenbrugge R (2007) Estimating the number of stroke patients eligible for thrombolytic treatment if delay could be avoided. Cerebrovasc Dis 23: 294-298.

2. Wardlaw JM, Murray V, Berge E, del Zoppo G, Sandercock P, et al. (2012) Recombinant tissue plasminogen activator for acute ischaemic stroke: an updated systematic review and meta-analysis. Lancet 379: 2364-2372.

3. Bhatia R, Hill MD, Shobha N, Menon B, Bal S, et al. (2010) Low rates of acute recanalization with intravenous recombinant tissue plasminogen activator in ischemic stroke: real-world experience and a call for action. Stroke 41: 2254-2258.

4. IMS Study Investigators (2004) Combined intravenous and intra-arteria recanalization for acute ischemic stroke: the Interventional Management of Stroke Study. Stroke 35: 904-911.

5. Hassan AE, Chaudhry SA, Grigoryan M, Tekle WG, Qureshi Al (2012) National trends in utilization and outcomes of endovascular treatment of acute ischemic stroke patients in the mechanical thrombectomy era. Stroke 43: 3012-3017.

6. Jellish WS, Edelstein SB (2015) General Anesthesia versus Conscious Sedation for the Endovascular Treatment of Acute Ischemic Stroke. J Stroke Cerebrovasc Dis 24: 1957-1960.

7. Balasubramaian A, Mitchell P, Dowling R, Yan B (2015) Evolution of endovascular therapy in acute stroke: implications of device development. J Stroke 17: 127-137.

8. Campbell BC, Mitchell PJ, Kleinig TJ, Dewey HM, Churilov L, et al. (2015) Endovascular therapy for ischemic stroke with perfusion-imaging selection. $\mathrm{N}$ Engl J Med 372: 1009-1018.

9. Saver JL, Goyal M, Bonafe A, Diener HC, Levy El, et al. (2015) Stent-retriever thrombectomy after intravenous t-PA vs. t-PA alone in stroke. N Engl J Med 372: 2285-2295.

10. Broderick JP, Palesch YY, Demchuk AM, Yeatts SD, Khatri $P$, et al. (2013) Endovascular therapy after intravenous t-PA versus t-PA alone for stroke. N Engl J Med 368: 893-903.

11. Kidwell CS, Jahan R, Gornbein J, Alger JR, Nenov V, et al. (2013) A trial of imaging selection and endovascular treatment for ischemic stroke. $\mathrm{N}$ Engl J Med 368: 914-923.

12. Macleod MR, Davis SM, Mitchell PJ, Gerraty RP, Fitt G, et al. (2005) Results of a multicentre, randomised controlled trial of intra-arterial urokinase in the treatment of acute posterior circulation ischaemic stroke. Cerebrovasc Dis 20: 12-17.

13. Bracard S, Ducrocq X (2015) THRACE: Trial and cost effectiveness evaluation of intra-arterial thrombectomy in acute ischemic stroke. In: European stroke organisation (ESO) conference, UK.

14. Goyal M, Demchuk AM, Menon BK, Eesa M, Rempel JL, et al. (2015) Randomized assessment of rapid endovascular treatment of ischemic stroke. N Engl J Med 372: 1019-1030.

15. Jovin TG, Chamorro A, Cobo E, de Miquel MA, Molina CA, et al. (2015) Thrombectomy within 8 hours after symptom onset in ischemic stroke. N Engl J Med 372: 2296-2306.

16. O'Rourke K, Berge E, Walsh CD, Kelly PJ (2010) Percutaneous vascula interventions for acute ischaemic stroke. Cochrane Database Syst Rev CD007574.

17. Fields JD, Khatri P, Nesbit GM, Liu KC, Barnwell SL, et al. (2011) Meta-analysis of randomized intra-arterial thrombolytic trials for the treatment of acute stroke due to middle cerebral artery occlusion. J Neurointerv Surg 3: 151-155. 
Citation: Cerrone P, Marini C (2016) Endovascular Treatment over Standard Medical Therapy in Acute Ischemic Stroke: A Meta-Analysis of Randomized Controlled Trials. Cardiovasc Pharm Open Access 5: 181. doi:10.4172/2329-6607.1000181

18. Fargen KM, Neal D, Fiorella DJ, Turk AS, Froehler M, et al. (2015) A metaanalysis of prospective randomized controlled trials evaluating endovascular therapies for acute ischemic stroke. J Neurolntervent Surg 7: 84-89.

19. Falk-Delgado A, Kuntze Söderqvist Å, Fransén J, Falk-Delgado A (2015) Improved clinical outcome 3 months after endovascular treatment, including thrombectomy, in patients with acute ischemic stroke: a meta-analysis. J Neurointervent Surg.

20. Chen CJ, Ding D, Starke RM, Mehndiratta P, Crowley RW, et al. (2015) Endovascular vs. medical management of acute ischemic stroke. Neurology 85: $1980-1990$

21. Hong KS, Ko SB, Lee JS, Yu KH, Rha JH (2015) Endovascular Recanalization Therapy in Acute Ischemic Stroke: Updated Meta-analysis of Randomized Controlled Trials. J Stroke 17: 268-281.

22. Ciccone A, Valvassori L, Ponzio M, Ballabio E, Gasparotti R, et al. (2010) Intra-arterial or intravenous thrombolysis for acute ischemic stroke? The SYNTHESIS pilot trial. J Neurointerv Surg 2: 74-79.

23. Ciccone A, Valvassori L, Nichelatti M, Sgoifo A, Ponzio M, et al. (2013) Endovascular treatment for acute ischemic stroke. N Engl J Med 368: 904-913.

24. Ducrocq X, Bracard S, Taillandier L, Anxionnat R, Lacour JC, et al. (2005) Comparison of intravenous and intra-arterial urokinase thrombolysis for acute ischaemic stroke. J Neuroradiol 32: 26-32.
25. Del Zoppo GJ, Higashida RT, Furlan AJ, Pessin MS, Rowley HA et al. (1998) PROACT: A phase II randomized trial of recombinant pro-urokinase by direct arterial delivery in acute middle cerebral artery stroke. Stroke 29: 4-11.

26. Furlan A, Higashida R, Wechsler L, Gent M, Rowley H, et al. (1999) Intraarterial prourokinase for acute ischemic stroke. The PROACT II study: A randomized controlled trial. Prolyse in Acute Cerebral Thromboembolism. JAMA 282: 2003-2011.

27. Ogawa A, Mori E, Minematsu K, Taki W, Takahashi A, et al. (2007) Randomized trial of intra-arterial infusion of urokinase within $6 \mathrm{~h}$ of middle cerebral artery stroke. Stroke 38: 2633-2639.

28. Berkhemer OA, Fransen PS, Beumer D, van den Berg LA, Lingsma HF, et al (2015) A randomized trial of intraarterial treatment for acute ischemic stroke. N Engl J Med 372: 11-20.

29. Mokin M, Kan P, Kass-Hout T, Abla AA, Dumont TM, et al. (2012) Intracerebral hemorrhage secondary to intravenous and endovascular intra-arterial revascularization therapies in acute ischemic stroke: an update on risk factors, predictors, and management. Neurosurg Focus 32. 